\title{
DISTRESS IN PREGNANT WOMEN
}

\author{
Ekaterina A. Burina, Valentin A. Ababkov, Elena A. Pazaratskas, \& Sofia V. Kapranova \\ St. Petersburg State University (Russia)
}

\begin{abstract}
Pregnancy is the most important stage in a woman's life, accompanied by the need to adapt not only at the physiological, but also psychological level. In this study, pregnancy is considered as a macro stress event that carries a certain stress load with different outcomes. The study reveals the specifics of distress at women in the dynamics: during pregnancy and after childbirth. Data was collected in antenatal clinic No.33 of the city of St. Petersburg, Russia. The total of 86 women were enrolled, aged 18 to 43 years $(M=29.47)$. The normal course of pregnancy was observed in 84 women. The methods based on transactional theory of stress were used for realization of scientific tasks. When analyzing the data obtained in the study, standard mathematical statistics methods were used using the computer programs "Excel" and "R Studio". It was found that a pregnancy of modern women is accompanied by lasting distress. Not only personal but also environmental factors take part in distress formation. Results testify statistically significant higher level of distress during pregnancy in contrast to a period after childbirth. Correlation analysis showed that the level of perceived stress correlates at a high significance level with each scale of the symptomatic questionnaire. Perceived stress is also associated with the coping strategy "Avoidance" and with the personality trait "Emotional stability", with an inverse correlation. The identification of psychological factors of distress during pregnancy indicates the need for psychological interventions to accompany pregnancy. The study is supported by the RFBR grant № 19-013-00417a.
\end{abstract}

Keywords: Pregnancy, distress, personality traits, perceived stress, coping-strategies.

\section{Introduction}

Pregnancy is the most important stage in a woman's life, accompanied by the need to adapt not only at the physiological, but also psychological level. In this study, pregnancy is considered not as a general psychological condition associated with psychopathology, but as a macro stress event that carries a certain stress load with different outcomes. Pregnancy is a special long-term stress, therefore, many women can experience various symptoms, mainly of a neurotic range. Usually, pregnancy support is carried out at the level of antenatal clinics and other special medical centers, that is, at the clinical level. There is definitely a need for psychological support, including the necessary intervention, which at present is clearly not enough. Solving this problem can help to improve mental and physical health of both women and their offspring (Perrez M., Baumann U., 2000).

The stress state of a woman during pregnancy is a predictor of emotional and cognitive difficulties, including the likelihood of a child developing attention deficit and hyperactivity disorder, as well as speech development delay. Prenatal stress in the mother affects the predisposition to diseases during the first year of life, which persists until the child is 6 years old (Zijlmans M.A. et al, 2017).

\section{Design, methods and sample}

The research goal is to study the characteristics and level of distress in pregnant women. This, among other factors, can contribute to the development of effective preventing and psychocorrectional measures aimed at reducing the distress severity in pregnant women.

The research design involves a longitudinal study with several measurements: when a pregnant woman is registered in the antenatal clinic; in the period from 2 to 6 months after delivery (with all respondents). 
To implement the research goal, the following methods were selected (based on the transactional theory of stress): 1. Clinical and psychological method (observation and conversation). 2. Clinical method (obstetric-gynecological: implemented by doctors). 3. Interview for collecting socio-demographic information. 4. Questionnaire "Scale of perceived stress-10". 5. The methodology "Methods of coping behavior". 6. Symptomatic questionnaire SCL-90. 7. The personal technique "Big Five". 8. "Pregnant relationship test".

When analyzing the data obtained in the study, standard mathematical statistics methods were used using the computer programs "Excel" and "R Studio", the packages "ez", "psych", and "ggplot2".

Data was collected in antenatal clinic No.33 of the city of St. Petersburg, Russia. The total of 86 women were enrolled, aged 18 to 43 years $(M=29.47)$, 85 were married (for 70 it was their first marriage), 43 had higher education, all women worked in different spheres, the income level per family member was average for this population. The normal course of pregnancy (without toxicosis) was observed in 84 women. Mental disorders in the respondents were not identified.

\section{Results and discussion}

The personality features of the respondents were studied. The results represent the general distribution of personality traits in this group of subjects.

The results of the "Pregnancy relationship test" showed a lack of a single determinant of attitude towards pregnancy. Most respondents are dominated by a combination of optimal and euphoric types. Depressive type was not found in the current sample.

The analysis of the results obtained for the symptomatic questionnaire showed the differences between the measurements are significant on each scale: $p<0.0001$. The results of the first measurement showed that the values on all scales of the symptomatic questionnaire fall into the range of high indicators when compared with the norm. The results of the second measurement demonstrate a statistically significant decrease in the performance of all scales of the symptomatic questionnaire, which at this stage are in the range of average values. Attention is drawn to a high level of general severity in the first measurement, which may indicate an adverse effect of the experience and course of pregnancy.

The general level of stress perceived by respondents was studied. The results of the initial measurement demonstrate a significantly $(\mathrm{p}<0.0001)$ higher level of stress $(M=25.05 \pm 6.86)$ than the indicators obtained in the following measurement $(\mathrm{M}=23.43 \pm 5.06)$.

The respondents' coping strategies used cover the entire possible range at a moderate level of severity. An analysis of the data obtained using the "Methods of coping behavior" methodology shows statistically significant changes for all coping strategies, with the exception of the self-control strategy, which does not endure any changes and is at the level of the first measurement. These data should be taken into account when planning and conducting psychological interventions in pregnant women.

A correlation analysis, revealing a large number of connections, was carried out. In particular, the level of perceived stress correlates at a high level of significance $(p<0.0001)$ with each scale of the symptomatic questionnaire, with the exception of the "Phobia" scale. Moreover, perceived stress is associated with such coping behavior strategy as "Avoidance" $(\mathrm{r}=0.530 ; \mathrm{p}=0.000)$ and with a personality trait "Emotional stability", with the opposite correlation $(r=-0.730 ; p=0.000)$.

The relationship between the age of respondents and all other parameters studied were also observed with no significant results found. An analysis of the relationship between gestational age and the studied clinical and psychological features also did not demonstrate any significant connections.

\section{Conclusions}

In conclusion, it can be noted that such significant changes in the state of respondents in dynamics are due primarily to successful births with an improvement in their physical and psychological state. Moreover, it can be assumed that a change in physical condition after childbirth actualizes the contrast effect. In general, the results justify and confirm the feasibility of using the mentioned theoretical approach (transactional theory of stress) to the phenomenon of pregnancy in general.

According to the study results, it is possible to develop the program of psychocorrectional and preventing measures, which should be implemented during pregnancy in order to improve the condition of women.

The study is supported by the RFBR grant № 19-013-00417a. 


\section{References}

Perrez M., Baumann U. Lehrbuch Klinische Psychologie - Psychotherapie. 3., vollstandig uberarbeitete Auflage. Bern: Verlag Hans Huber. 2005. Psychology. 2000. № 19 (6). P. 544-553.

Zijlmans M.A., Beijers R., Riksen-Walraven M.J., de Weerth C. Maternal late pregnancy anxiety and stress is associated with children's health: a longitudinal study. Stress. 2017, Sep; 20 (5): 495-504. 